\title{
Results of the searches for pentaquarks with strangeness in DIS at HERA
}

\author{
Sergei Chekanov ${ }^{* \dagger}$ \\ HEP division, Argonne National Laboratory, 9700 S.Cass Avenue, Argonne, IL 60439, USA \\ E-mail: chekanov@mail.desy.de
}

The $K_{S}^{0} p$ invariant mass spectrum was reconstructed in several kinematic regions with the main emphasis on the studies of the production mechanism of the $\Theta^{+}$candidate recently observed by ZEUS. The candidate $\Theta^{+}$signal was found to be produced predominantly in the forward hemisphere in the laboratory frame. This is unlike the case for the $\Lambda(1520)$ or the $\Lambda_{c}$, and indicates that the $\Theta^{+}$may have an unusual production mechanism related to proton-remnant fragmentation. H1 does not observe a signal and sets an upper limit at 95\% C.L. which does not exclude the ZEUS observation.

International Europhysics Conference on High Energy Physics

July 21st - 27th 2005

Lisboa, Portugal

\footnotetext{
*Speaker.

${ }^{\dagger}$ for the $\mathrm{H} 1$ and ZEUS Collaborations
} 


\section{Introduction}

Recently, ZEUS made an observation [1] of a narrow signal in the vicinity of $1530 \mathrm{MeV}$ which can be interpreted as a bound state of five quarks, i.e. as a pentaquark, $\Theta^{+}=u u d d \bar{s}$ [2]. The experimental search was performed using inclusive ep collisions with the proton energy 820/920 $\mathrm{GeV}$ and the electron/positron energy of $27.6 \mathrm{GeV}$. The data were collected using an integrated luminosity of $121 \mathrm{pb}^{-1}$ from HERA I.

The $\Theta^{+}$candidate decaying to $K_{S}^{0} p(\bar{p})$ was seen in deep inelastic scattering (DIS) at $Q^{2}>$ $20 \mathrm{GeV}^{2}$, while no signal was reported in photoproduction $\left(Q^{2} \sim 0\right)$. This decay channel is not exotic since 3-quark states, such as $\Sigma$ baryons, can also decay to the $K_{S}^{0} p(\bar{p})$. Therefore, it is essential to study the kinematics of the observed signal and to compare it with that for established 3-quark states.

The absence of a guiding principle of how to compose three quarks to form a baryon at the quark-fragmentation stage leads to a few possible baryon-production mechanisms. In the case of baryons with five quarks, the situation could be even more complicated. For collisions involving incoming baryon(s), the fragmentation mechanism could be influenced by a contribution from the proton-remnant system, thus the baryonic yield in certain kinematic regions of $e p$ collisions is expected be higher than in $e^{+} e^{-}$annihilation.

\section{Evidence for a baryonic state near 1530 decaying to $K_{S}^{0} p$}

$K_{S}^{0} p(\bar{p})$ invariant masses reconstructed in photoproduction and DIS using HERA I data are shown in Fig. 1(left). The $K_{S}^{0} p(\bar{p})$ distribution has two peaks, at around $1522 \mathrm{MeV}\left(Q^{2}>20 \mathrm{GeV}^{2}\right)$ and near $2286 \mathrm{MeV}$. The first peak is attributed to the $\Theta^{+}$candidate state [1]. The second peak corresponds to the established $\Lambda_{c}$ baryon. Both peaks are best seen for $Q^{2}>20 \mathrm{GeV}^{2}$, i.e. in the region where the $\Lambda_{c}$ peak has the largest signal-over-background ratio ( $\left.\mathrm{S} / \mathrm{B}=0.22\right)$. Since the average charged-track multiplicity is $50 \%$ higher $^{1}$ for photoproduction than for DIS at $Q^{2}>1 \mathrm{GeV}^{2}$, the $\mathrm{S} / \mathrm{B}$ ratio is small for photoproduction. Therefore, the significant combinatorial background for the $K_{S}^{0} p(\bar{p})$ spectrum could explain the non-observation of the $\Theta^{+}$candidate for photoproduction [1].

It is important to note that the decrease of the $\mathrm{S} / \mathrm{B}$ ratio for high-multiplicity events exists only for the states whose production is not driven by pure quark fragmentation. A typical example is $\Lambda_{c}$ : there could be at most two $\Lambda_{c}$ per event independent of the energy contributing to the hadronicfinal state. The production of charmed quarks is significantly suppressed at the fragmentation stage, therefore, a hard QCD subprocess, like $\gamma^{*} g \rightarrow c \bar{c}$, is the necessary mechanism for the $\Lambda_{c}$ formation. If the $\Theta^{+}$production is driven by the diquark system (in which the diquark is produced by the incoming proton), then the $\Theta^{+}$peak should also have a small S/B ratio for high multiplicity events.

In contrast, the S/B ratio for $\Lambda(1520)$ reported by ZEUS [3] is almost the same for photoproduction and DIS. This is a clear indication that $\Lambda(1520)$ is solely produced by the quark fragmentation, and, therefore, this baryon cannot be used as a reference state for experimental $\Theta^{+}$ searches.

\footnotetext{
${ }^{1}$ This is due to the fact that photoproduction events were taken using a specific trigger requirement (jets with $\left.E_{T}>6-8 \mathrm{GeV}\right)$. The hadronic-fi nal state of DIS data sample is almost completely trigger-unbiased.
} 
ZEUS
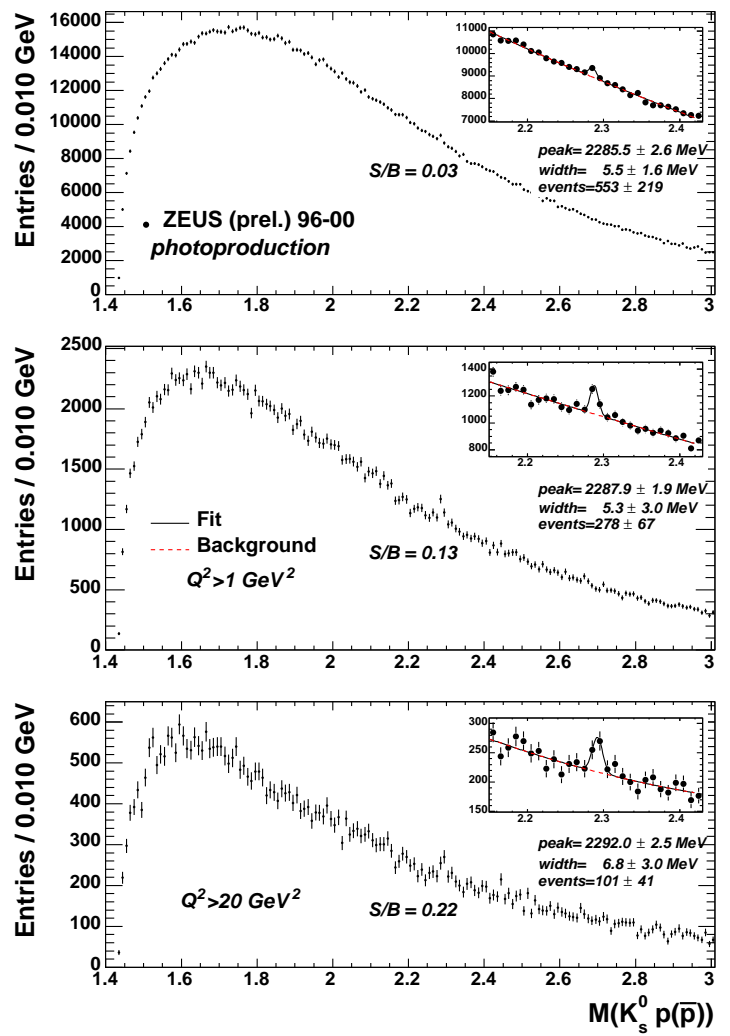

ZEUS
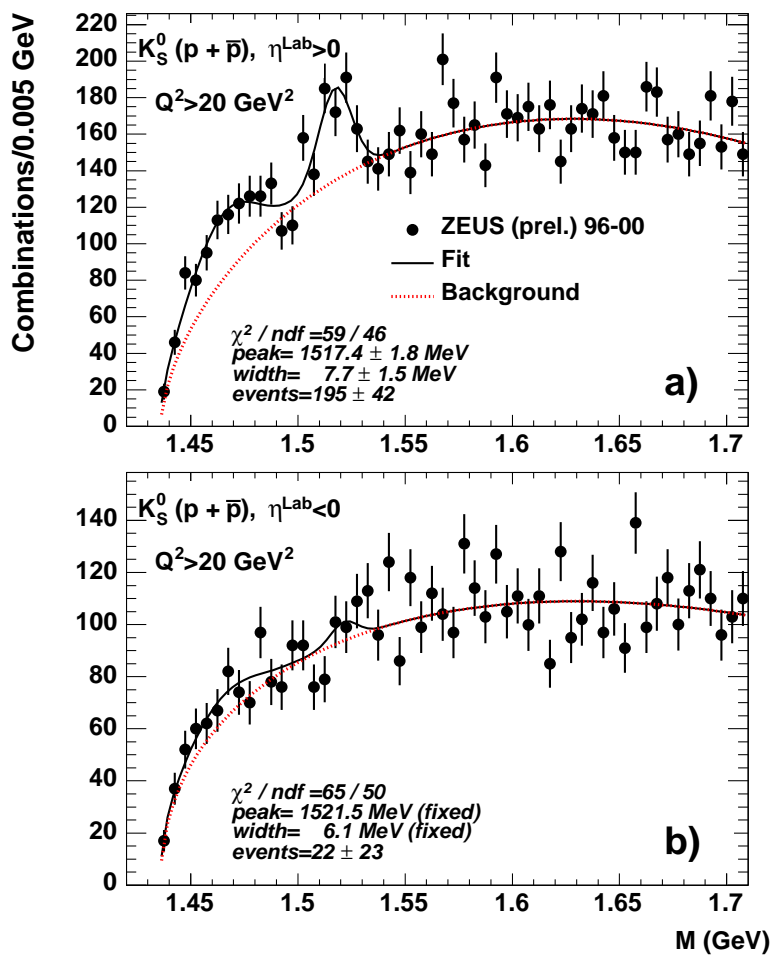

Figure 1. Left: $K_{S}^{0} p(\bar{p})$ invariant-mass spectra in photoproduction and DIS $\left(Q^{2}>1 \mathrm{GeV}^{2}\right.$ and $\left.Q^{2}>20 \mathrm{GeV}^{2}\right)$ with the indicated signal-over-background ratio $(S / B)$ for the $\Lambda_{c}$ peak. The insets show the invariant-mass distribution near the $\Lambda_{c}$ mass region. Right: $K_{S}^{0} p(\bar{p})$ invariant-mass distribution near the $1520 \mathrm{MeV}$ mass for the forward $(\eta>0)$ and rear $(\eta<0)$ region. The fit was performed using a double Gaussian with the threshold function.

If the observed $K_{S}^{0} p(\bar{p})$ peak near $1522 \mathrm{MeV}$ indeed corresponds to a new baryonic state, then the studies of this peak in different pseudorapidity regions can help to understand the production mechanism of this state. The $1522 \mathrm{MeV}$ peak should be seen for both forward and rear pseudorapidity regions if the $\Theta^{+}$is produced by pure quark fragmentation, as any established 3quark state with strangeness. In contrast, if the production mechanism of the $\Theta^{+}$state involves the fragmentation of the diquark system from the incoming proton, then the $\Theta^{+}$state should mainly be seen for $\eta>0$ (towards the incoming proton direction) and at low transverse momenta. In addition, high $Q^{2}$ DIS is more favorable [4] as in this case the incoming proton can receive a sufficient kick from the virtual boson and the color string can drag the diquark towards the central pseudorapidity region (small $\eta$ ). Indeed, Fig. 1(right) shows that the $1522 \mathrm{MeV}$ signal is found to occur predominantly for $\eta>0$. Known baryons, produced either via boson-gluon mechanism $\left(\Lambda_{c}\right)$ or by quark-fragmentation process $(\Lambda(1520))$, are distributed uniformly over the whole measured pseudorapidity region $-1.5<\eta<1.5$ (see Fig. 2). 

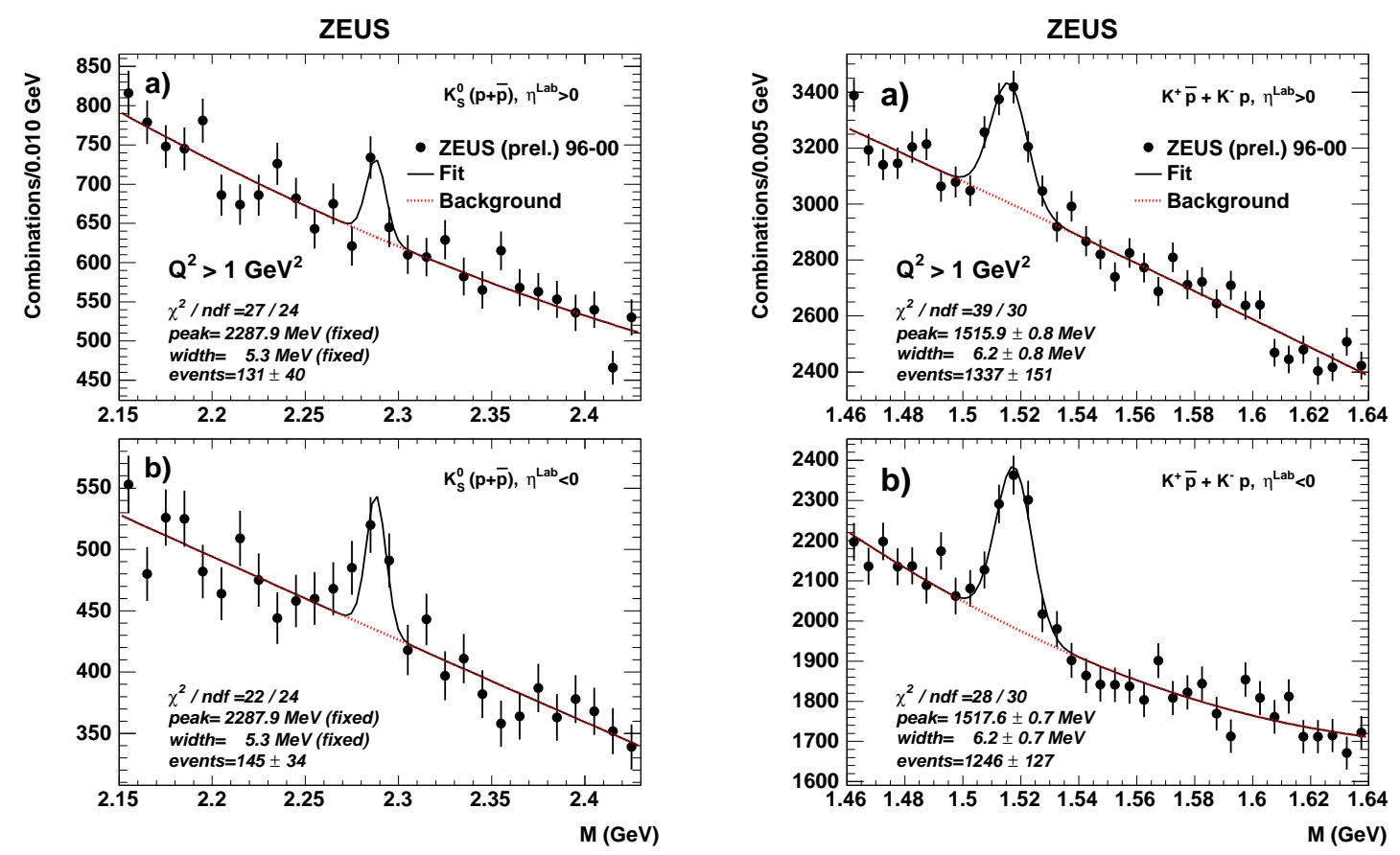

Figure 2. Left: $K_{S}^{0} p(\bar{p})$ invariant-mass distribution near the $\Lambda_{c}$ mass for $\eta>0$ and $\eta<0$ regions for $Q^{2}>1 \mathrm{GeV}^{2}$. The peak position and the width were fixed from the fit to the sum of these two mass distributions. Right: The $K^{-} p\left(K^{+} \bar{p}\right)$ invariant-mass distribution near the $\Lambda(1520)$ mass in the forward $(\eta>0)$ and in the rear $(\eta<0)$ regions for $Q^{2}>1 \mathrm{GeV}^{2}$. The fits were performed using a Gaussian with a second-order polynomial function.

\section{H1 results}

The H1 Collaboration has performed $\Theta^{+}$searches using a similar $K_{S}^{0} p(\bar{p})$ reconstruction procedure [5] as for the ZEUS analysis, but the DIS selection was somewhat tighter than in the ZEUS case. An integrated luminosity of $71 \mathrm{pb}^{-1}$ was used from HERA I. The $K_{S}^{0} p(\bar{p})$ spectrum is shown in Fig. 3(left). No signal is observed. Given the absence of the $\Theta^{+}$signal, 95\% C.L. limits were set on the production of new states decaying to $K_{S}^{0} p(\bar{p})$ in the mass range 1480-1700 $\mathrm{MeV}$ in DIS for $Q^{2}>20 \mathrm{GeV}^{2}$. The limits shown in Fig. 3(right) were obtained assuming that the production kinematics of $\Theta^{+}$is similar to the $\Sigma^{+}$state produced by pure quark-fragmentation process. Around the mass of $1520 \mathrm{MeV}$, an upper limit on the cross section of roughly 100-120 pb was found, which is about the signal cross section observed ${ }^{2}$ by ZEUS.

In addition, $\mathrm{H} 1$ investigated the $K_{S}^{0} p(\bar{p})$ invariant masses at lower $Q^{2}$, using an alternative selection which uses higher-momentum protons, $p>1.5 \mathrm{GeV}$. In this case, a lower proton purity is expected. The $K_{S}^{0} p$ and $K_{S}^{0} \bar{p}$ mass combinations were analysed separately. As for the ZEUS studies [1], a $\Theta^{+}$signal was not seen at low $Q^{2}$ and for high-momentum protons.

\footnotetext{
${ }^{2}$ The ZEUS cross section [6] for the $\Theta^{+}$candidates and their antiparticles measured in the kinematic region given by $Q^{2} \geq 20 \mathrm{GeV}^{2}, 0.04<y<0.95, p_{T}>0.5 \mathrm{GeV}$ and $|\eta|<1.5$ was $\sigma\left(e^{ \pm} p \rightarrow e^{ \pm} \Theta^{+} X \rightarrow e^{ \pm} K^{0} p X\right)=125 \pm$ 27 (stat. $)_{-28}^{+36}$ (syst.) pb
} 

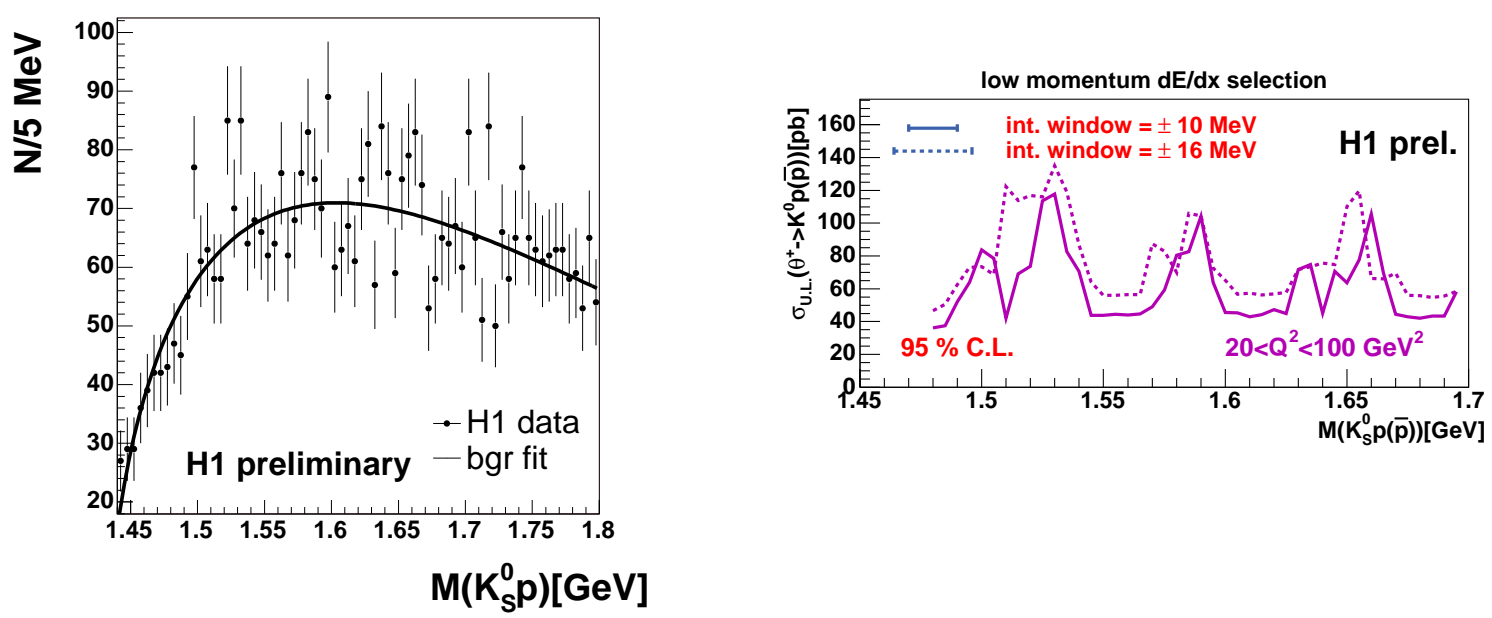

Figure 3. Left: $K_{S}^{0} p(\bar{p})$ invariant-mass distribution near the $1520 \mathrm{MeV}$ mass in DIS for $Q^{2}>$ $20 \mathrm{GeV}^{2}$ reconstructed by H1. Right: Upper limits on the cross section $\sigma\left(e^{ \pm} p \rightarrow e^{ \pm} \Theta^{+} X \rightarrow\right.$ $\left.e^{ \pm} K^{0} p X\right)$.

\section{4. $\Xi_{3 / 2}^{--}$and $\Xi_{3 / 2}^{0}$ states?}

The $\Theta^{+}$lies at the apex of a hypothetical anti-decuplet of pentaquarks with spin $1 / 2$ [2]. The baryonic states $\Xi_{3 / 2}^{--}$and $\Xi_{3 / 2}^{0}$ at the bottom of this antidecuplet are also manifestly exotic. A support for this picture came from the NA49 experiment which recently made observations [7] of both states near $1862 \mathrm{MeV}$ in the $\Xi^{-} \pi^{ \pm}$and $\bar{\Xi}^{+} \pi^{ \pm}$decay channels in fixed-target $p p$ collisions at the CERN SPS.

ZEUS has performed a similar analysis [8] by combining $\Lambda$ with $\pi$ using displaced tertiary vertices. With more than 190 reconstructed $\Xi^{0}(1530)$ near the mass threshold of $\Xi \pi$ spectrum, no pentaquark signal was observed near the $1860 \mathrm{MeV}$ mass region. The 95\% C.L. upper limit on the ratio $\Xi_{3 / 2}^{--}\left(\Xi_{3 / 2}^{0}\right)$ to $\Xi^{0}(1530)$ was in the range $0.2-0.45$.

\section{References}

[1] ZEUS Collaboration, S. Chekanov et al., Phys. Lett. B 591 (2004) 7.

[2] D. Diakonov, V. Petrov and M.V. Polyakov, Z. Phys. A 359 (1997) 305.

[3] ZEUS Collaboration, Contributed paper to EPS05, abs 277,369, Study of production properties of baryons decaying to strange particles in ep collisions at HERA

[4] S. Chekanov, Preprint hep-ph/0502098, (Eur. J. Phys. C, in press)

[5] H1 Collaboration, Contributed paper to EPS05, abs 400, Search for a narrow baryonic resonance decaying to $K_{S}^{0} p\left({ }^{-} p\right)$

[6] ZEUS Collaboration, Contributed paper to EPS05, abs 290,392, Cross section measurements of a narrow baryonic state decaying to $K_{S}^{0} p\left({ }^{-} p\right)$ in deep inelastic scattering at HERA

[7] NA49 Collaboration, C. Alt et al., Phys. Rev. Lett. 92 (2004) 042003.

[8] ZEUS Collaboration, S. Chekanov et al., Phys. Lett. B 610 (2005) 212. 